\title{
Polyanionic carbohydrate doxorubicin-dextran nanocomplex as a delivery system for anticancer drugs: in vitro analysis and evaluations
}

This article was published in the following Dove Press journal:

International Journal of Nanomedicine

8 July 2011

Number of times this article has been viewed

\author{
Parisa Yousefpour ${ }^{1,2}$ \\ Fatemeh Atyabi' \\ Ebrahim Vashegani Farahani ${ }^{3}$ \\ Ramin Sakhtianchi' \\ Rassoul Dinarvand' \\ 'Nanotechnology Research Center, \\ Faculty of Pharmacy, Tehran University \\ of Medical Sciences, Tehran, Iran; \\ ${ }^{2}$ Department of Biotechnology, \\ Faculty of Science, University of \\ Tehran, Tehran, Iran ${ }^{3}$ Department of \\ Chemical Engineering, Tarbiat Modares \\ University, Tehran, Iran
}

Correspondence: Fatemeh Atyabi

Nanotechnology Research Center, Faculty of Pharmacy, Tehran University of Medical Sciences, Tehran, I4I76I44| I, Iran

Tel +982166959052

Fax +982166959052

Email atyabifa@tums.ac.ir

\begin{abstract}
This study deals with the preparation and investigation of a nanoscale delivery system for the anticancer drug doxorubicin (DOX) using its complexation with polyanionic carbohydrate dextran sulfate (DS). Dynamic light scattering, SEM, and zeta potential determination were used to characterize nanocomplexes. DOX-DS complexation was studied in the presence of ethanol as a hydrogen-bond disrupting agent, $\mathrm{NaCl}$ as an electrostatic shielding agent, and chitosan as a positively charged polymer. Thermodynamics of DOX-DS interaction was studied using isothermal titration calorimetry (ITC). A dialysis method was applied to investigate the release profile of DOX from DOX-DS nanocomplexes. Spherical and smooth-surfaced DOX-DS nanocomplexes $(250-500 \mathrm{~nm})$ with negative zeta potential were formed at a DS/DOX $(\mathrm{w} / \mathrm{w})$ ratio of $0.4-0.6$, with over $90 \%$ drug encapsulation efficiency. DOX when complexed with DS showed lower fluorescence emission and $480 \mathrm{~nm}$ absorbance plus a $15 \mathrm{~nm}$ bathometric shift in its visible absorbance spectrum. Electrostatic hydrogen bonding and $\pi-\pi$ stacking interactions are the main contributing interactions in DOX-DS complexation. Thermal analysis of DOX-DS complexation by ITC revealed that each DOX molecule binds with 3 DS glycosyl monomers. Drug release profile of nanocomplexes showed a fast DOX release followed by a slow sustained release, leading to release of $32 \%$ of entrapped DOX within 15 days. DOX-DS nanocomplexes may serve as a drug delivery system with efficient drug encapsulation and also may be taken into consideration in designing DOX controlled-release systems.
\end{abstract}

Keywords: chitosan, dextran, doxorubicin, nanocomplex, anticancer, drug delivery

\section{Introduction}

The anthracycline antibiotic doxorubicin (DOX) is a potent chemotherapeutic agent that has been used for over 30 years to treat a wide spectrum of human malignancies, especially breast cancer and lymphoma. ${ }^{1}$ However, despite its efficiency and wide use, its therapeutic utility is limited due to dose-limiting toxicities such as bone marrow suppression ${ }^{2}$ and congestive heart failure. ${ }^{3-5}$ Another challenge met by DOX is the development of resistance mechanisms by tumor cells, ${ }^{6-8}$ which reduce the intracellular drug concentration either by reducing drug uptake or enhancing drug efflux. ${ }^{9}$

Numerous studies have sought the solution to these problems in nanoparticulate carriers. ${ }^{10-19}$ Nanoscale drug carriers are intended to overcome drug resistance presumably by means of being enveloped in an endosome when entering the cell and thereby bypassing P-glycoprotein efflux pumps (one of the main drug-resistance mechanisms) and preventing drug efflux from the resistant tumors. ${ }^{20-23}$ One of the nano-oriented approaches undertaken to this end is the complexation of drugs with polymers. ${ }^{16}$ 
Electrostatic interaction can result in complexation between cationic drugs and anionic polymers. ${ }^{24,25}$ DOX possesses positive charge and has been studied for its complexation with various anionic polymers such as poly(acrylic acid), ${ }^{26,27}$ $\gamma$-polyglutamic acid, ${ }^{28}$ polyaspartate, ${ }^{24,29}$ polyglutamate, ${ }^{30}$ block ionomers of aspartate, benzyl aspartate, ${ }^{31-34}$ benzyl glutamate, ${ }^{29}$ and polyethylene oxide. These polyanions form polyelectrolyte complexes with DOX. Aromatic stacking and hydrogen bonding interactions are also assumed to play an important role in such complexes. ${ }^{26,27}$

Dextran sulfate (DS) is a highly anionic carbohydrate which has been investigated for improving encapsulation efficiency of DOX. Successful augmentation of DOX encapsulation into albumin microspheres was achieved with the aid of DS. ${ }^{35}$ Janes et al employed DS to increase DOX loading into chitosan nanoparticles. ${ }^{36}$ DOX has also been encapsulated into chitosan microparticles formulated via the complex coacervation method with DS. ${ }^{37}$ In a series of studies, DOX was conjugated with polymeric dextrans of various molecular weights and it was found that the cytotoxicity, and the DNA-binding kinetics of DOX were subsequently modified. ${ }^{38-40}$ Antitumor activity, acute toxicity, and plasma pharmacokinetics of DOX-DS conjugate were compared with free DOX in rats following iv administration. Lam et $\mathrm{al}^{41,42}$ showed that the DOX-DS conjugate exerted superior antitumor activity to the free DOX in multidrug-resistant cell lines, the reason being cited as a decrease in the removal rate of DOX from $\mathrm{P}$-glycoprotein overexpressing multidrugresistant cells once conjugated to dextran. Another group further investigated this possibility of overcoming the DOX resistance in vitro by using DOX-DS conjugate in a multidrug resistance subline of human carcinoma and found similar results. ${ }^{43}$ Herein, we report the formation of electrostatic complexes at nanoscale between positively charged DOX and negatively charged DS (DOX-DS). The experimental approach includes probing the nature of interactions in DOX-DS complexation, characterization of DOX-DS nanocomplexes, and release profile of drug molecules. DOX-DS interaction is also studied in the presence of the positively charged polymer chitosan.

\section{Materials and methods Materials}

DS (D4911, MW of 8000) was obtained from Sigma Chemical Co. Doxorubicin (purity $~ 98.5 \%$ ) was purchased from RPG Life Sciences Limited (Ankleshwar, India). All other chemicals were of analytical grade; deionized water was used throughout.

\section{Preparation and characterization of DOX-DS nanocomplexes}

DOX $\mathrm{HCl}$ was dissolved in deionized water at a concentration of $0.1 \mathrm{mg} / \mathrm{mL}$. To this solution, an equal volume of DS solution in deionized water was added to give final DOX-DS solutions of 0.1, 0.2, 0.3 0.4, 0.5, 0.6, 0.8, 1, 1.5, 2, 3, and $4 \mathrm{w} / \mathrm{w}$. Solutions were left for 45 minutes in the dark while stirring. The hydrodynamic mean diameter and zeta potential of DOX-DS nanocomplexes were measured by, respectively, dynamic light scattering and laser doppler electrophoresis using Zetasizer (Nano-ZS, Malvern, UK). All dynamic light scattering measurements were performed with a wavelength of $633 \mathrm{~nm}$ at $25^{\circ} \mathrm{C}$ with an angle detection of $90^{\circ}$. All measurements were run in triplicate.

\section{Analysis of encapsulation efficiency}

Encapsulation efficiency of DOX-DS nanocomplexes was analyzed by ultracentrifugation of complexes at $80,000 \mathrm{rpm}$ for 30 minutes. To make sure that free drug did not precipitate at that high rotation speed, free drug solution was used as control. The concentration of remaining DOX in supernatant was measured spectroscopically at $480 \mathrm{~nm}$ and encapsulation efficiency was determined by applying the following equation:

$$
\begin{aligned}
& \text { Encapsulation } \\
& \text { efficiency }(\%)
\end{aligned}=\frac{[D O X]_{\text {total }}-[D O X]_{\text {supernatant }}}{[D O X]_{\text {total }}} \times 100
$$

\section{Scanning electron microscopy (SEM)}

The morphological characteristics of nanoparticles were observed by using an SEM (XL 30, Philips, The Netherlands). Nanoparticle suspensions were spread on an aluminium disc and allowed to dry at room temperature. The dried nanoparticles were then coated with gold metal using a sputter coater (SCD 005, Bal-Tec, Switzerland).

\section{Spectrophotometry and fluorescence spectroscopic study of DOX-DS nanocomplexes}

DOX solutions of different concentrations were mixed with DS in ratios of $0.1: 1,0.2: 1,0.3: 1,0.4: 1,0.5: 1,0.6: 1,0.7: 1$, $0.8: 1,1: 1,1.5: 1,2: 1,3: 1$, and $4: 1$. The absorbance of the resulting nanocomplexes was measured at $480 \mathrm{~nm}$ using a UV-VIS spectrophotometer (Scinco S-3100, Korea). The fluorescence measurement was carried out on a fluorescence spectrophotometer (Cary Eclipse, Varian, Australia) equipped with a thermostatically controlled cell holder at an ambient temperature. The monochromatic slits were set at 
$5 \mathrm{~nm}$ to reduce the intensity of the signal depending on the experiment. The wavelength of excitation was $470 \mathrm{~nm}$ and the spectra were recorded between 500 and $700 \mathrm{~nm}$. Water was used as the baseline reference in both studies.

\section{DOX-DS interaction study}

To come to a deeper understanding of the nature of interactions involved, DOX-DS complexation was studied in presence of ethanol as a hydrogen-bond disrupting agent and $\mathrm{NaCl}$ as an electrostatic shielding agent. Ethanol was added to the DOX-DS nanocomplex solution (DOX: $51.7 \mu \mathrm{M}, \mathrm{DS} / \mathrm{DOX}: 0.6$ ) at different $\mathrm{v} / \mathrm{v}$ proportions and the absorbance at $480 \mathrm{~nm}$ was measured and compared with that of pure DOX in the corresponding ethanol-water cosolvent system. The absorbance of the DOX-DS formulation was also studied in different ionic strengths, ie, different concentrations of $\mathrm{NaCl}$.

In addition, the absorbance of the DOX-DS system was examined in presence of chitosan (CS) as a positively charged polymer. CS was added to DOX-DS system (DOX: $51.7 \mu \mathrm{M}$, DS/DOX: 0.6) at CS/DS (w/w) ratio of 0.25, 0.5, 0.75, 1, 2, and 3; the resulting $480 \mathrm{~nm}$ absorbance was studied.

\section{In vitro evaluation of DOX release from nanocomplexes}

In vitro drug release profile of DOX-DS nanocomplexes was investigated as follows. Nanocomplex suspension (DOX $50 \mu \mathrm{g} / \mathrm{mL}, \mathrm{DS} / \mathrm{DOX}$ 0.6) was placed into dialysis tubing (Sigma dialyses tubes Mw cutoff $12 \mathrm{kDa}$ ) with phosphate buffer solution (PBS) as release medium. The nanocomplex was then incubated in a shaking water bath at $37^{\circ} \mathrm{C}$. At appropriate time intervals, a certain volume of the release medium was taken out and was replaced by the same volume of fresh PBS. Sampling volumes were selected to ensure sink conditions, ie, to maintain the drug concentration in dialysate below $10 \%$ of its aqueous solubility. The amount of DOX in the release medium was examined by HPLC. A solution of free DOX was also loaded into dialysis tubing and dialyzed under the same condition as control.

The cumulative fraction of released DOX was calculated according to the following equation:

Cumulative released DOX\% (tn)

$$
\begin{aligned}
& =\frac{\text { total released DOX }}{\text { initial DOX in nanocomplex formulation }} \times 100 \\
& =\frac{\sum_{\mathrm{i}=1}^{\mathrm{i}=\mathrm{n}-1}[\mathrm{DOX}]_{\mathrm{i}} \mathrm{V}_{\mathrm{i}}+[\mathrm{DOX}]_{\mathrm{f}} \mathrm{V}_{\mathrm{f}}}{[\mathrm{DOX}]_{0} \mathrm{~V}_{0}} \times 100
\end{aligned}
$$

where $[\mathrm{DOX}]_{\mathrm{i}}$ and $\mathrm{V}_{\mathrm{i}}$ represent the measured DOX concentration in and volume of sample taken at time $t_{i}$; $[D O X]_{f}$ and $V_{f}$, final measured DOX concentration in and final volume of release medium at time (tn); [DOX $]_{0}$ and $\mathrm{V}_{0}$, initial DOX concentration in and volume of the nanocomplex formulation under study.

In addition, DOX release rate $(\% / \mathrm{hr})$ was estimated as:

$$
\text { release rate }=\frac{d(\% D O X)}{d t}=\frac{(\% D O X)_{t_{n}}-(\% D O X)_{t_{n-1}}}{t_{n}-t_{n-1}}
$$

where $(\% \mathrm{DOX})_{\mathrm{t}_{\mathrm{n}}}$ and $(\% \mathrm{DOX})_{\mathrm{t}_{\mathrm{n}-1}}$ are percentage of released DOX at sampling time $\mathrm{t}_{\mathrm{n}}(\mathrm{hr})$ and $\mathrm{t}_{\mathrm{n}-1}(\mathrm{hr})$.

\section{HPLC analysis of DOX}

Released DOX in the dialysate was quantified by $\mathrm{C} 18$ reverse phase column connected to a Knauer HPLC system; the Knauer HPLC apparatus consisted of an S 1000 pump with fluorescence detector (RF 10 Axl). Mobile phase was composed of a mixture of $0.01 \mathrm{M}$ potassium dihydrogen phosphate and acetonitrile in a ratio of 65:35 whose final $\mathrm{pH}$ was adjusted to 4.2 using phosphoric acid. The flow rate was set to $1 \mathrm{~mL} /$ minute and the fluorescence (excitation $470 \mathrm{~nm}$, emission $550 \mathrm{~nm}$ ) was recorded. All determinations were carried out at ambient temperature. Under these conditions DOX showed a retention time of about 4 minutes. DOX concentrations were calculated with reference to a calibration curve.

\section{ITC study}

The isothermal titration microcalorimetric experiments were performed with ITC instrument (VP-ITC, MicroCal, LLC, Northampton, MA). The titration vessel was made from stainless steel. DS solution (4797 $\mu \mathrm{M}$ glycosyl unit) was injected by use of a Hamilton syringe into the calorimetric titration vessel, which contained $1.8 \mathrm{~mL}$ DOX $(862 \mu \mathrm{M})$. Thin (0.15 $\mathrm{mm}$ inner diameter) stainless steel hypodermic needles, permanently fixed to the syringe, reached directly into the calorimetric vessel. Injection of the DS solution into the perfusion vessel was repeated 20 times, with $10 \mu \mathrm{L}$ per injection. The calorimetric signal was measured by a digital voltmeter which was part of a computerized recording system. The heat of each injection was calculated by the Origin 7.0 software program (OriginLab Corp, Northampton, MA). The microcalorimeter was frequently calibrated electrically during the course of the study.

The ITC of study was also performed with DOX and DS solved in $0.15 \mathrm{M}$ aqueous solution of $\mathrm{NaCl}$ (ionic strength $0.15 \mathrm{M})$. Injections were carried out 20 times $(15 \mu \mathrm{L}$ each time). 


\section{Results}

\section{Characterization of nanocomplexes}

As illustrated in Table 1, mean hydrodynamic diameter, polydispersity index, and zeta potential of DOX-DS nanocomplexes containing DOX at the concentration of $0.05 \mathrm{mg} / \mathrm{mL}$ and different DS/DOX ratios were measured. Among different DS/DOX ratios examined, ratios between 0.4 and 0.6 led to nanocomplexes.

\section{Complexation efficiency}

The efficiency of DOX encapsulation by DOX-DS nanocomplexes was highly affected by DS/DOX ratio. DOX complexation was enhanced with increasing DS/DOX ratio until it reached a peak of $95 \%$ at a DS/DOX ratio of 0.5 . Efficiency then declined with further increments in the DS/DOX ratio (Figure 1).

\section{Surface morphology of nanocomplexes}

Figure 2A clearly shows regular smooth and spherical nanocomplexes free from aggregation.

\section{Absorbance and florescence spectrum of DOX-DS nanocomplexes}

Absorbance spectrum of DOX in water shows a maximum at $480 \mathrm{~nm}$ and 2 shoulders at 500 and $535 \mathrm{~nm}$. Complexation with DS as depicted in Figure 2B caused a bathochromic shift to $\lambda_{\text {max }} 495 \mathrm{~nm}$, and a hypochromic and broadened spectrum. No shift was observed in the florescence emission spectrum, but the intensity was lowered more drastically compared with the visible absorbance spectrum (Figure 2C).

The relative decrease in $480 \mathrm{~nm}$ absorbance of DOX-DS complexes with respect to free DOX was independent of DOX concentration and dependent on DS/DOX ratios. As shown in Figure 3, absorbance decreased with further addition of DS until a plateau $\left(A_{480}^{D O X-D S} / A_{480}^{D O X}=0.68\right)$ was achieved at DS/DOX ratio of 0.4 , after which no further decrease in the above absorbance fraction was observed.

\section{DOX-DS interaction}

To investigate the role played by hydrogen bond and electrostatic interaction in DOX-DS complexation, such

Table I Mean hydrodynamic diameter, polydispersity index (PDI), and zeta potential of DOX-DS complexes containing DOX at the concentration of $0.05 \mathrm{mg} / \mathrm{mL}$ and different DS/DOX ratios

\begin{tabular}{llll}
\hline $\begin{array}{l}\text { DS/DOX } \\
\text { ratio }(\mathbf{w} / w)\end{array}$ & $\begin{array}{l}\text { Mean hydrodynamic } \\
\text { diameter }(\mathrm{nm})\end{array}$ & PDI & $\begin{array}{l}\text { Zeta } \\
\text { potential }(\mathbf{m V})\end{array}$ \\
\hline $4: 10$ & $461 \pm 31$ & $0.257 \pm 0.061$ & $-19.5 \pm 1.4$ \\
$6: 10$ & $279 \pm 28$ & $0.29 \pm 0.014$ & $-33.2 \pm 3.3$ \\
\hline
\end{tabular}

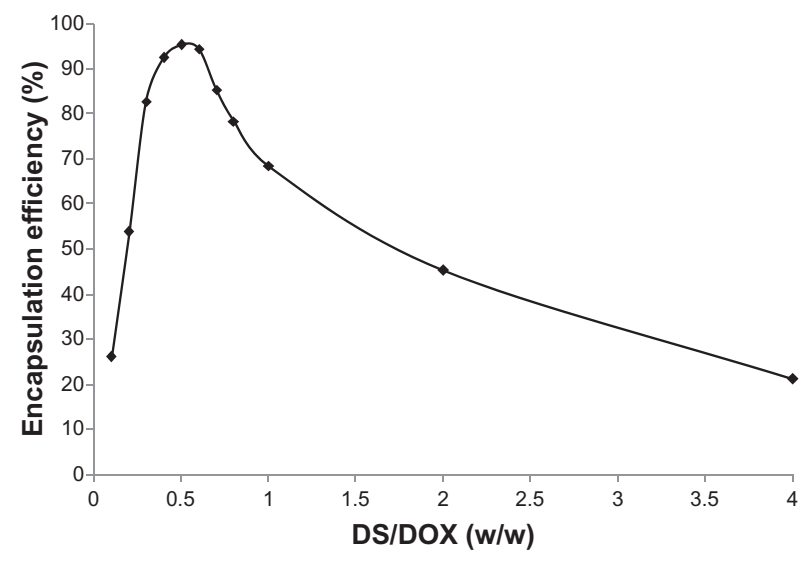

Figure I Effect of dextran sulfate/doxorubicin (DS/DOX) ratio on complexation efficiency of DOX-DS complexes.

interactions between DOX and DS were inhibited by the addition of ethanol and $\mathrm{NaCl}$ and the corresponding absorbance was measured. The results revealed that in presence of either agent, the absorbance increased; moreover, at concentrations $>50 \% \mathrm{v} / \mathrm{v}$ ethanol or $0.3 \mathrm{M} \mathrm{NaCl}$, the absorbance of DOX cosolved with DS was equal to that of free drug (Figures 4A and 4B).

Moreover, polycationic carbohydrate was demonstrated to influence DOX-DS interaction as well. Figure 4C shows that the presence of CS up to CS/DS (w/w) 0.5 caused a slight decrease in DOX-DS absorbance. Higher CS/DS (w/w), however, increased DOX-DS absorbance, until at CS/DS $(w / w)>3$, DOX-DS absorbance reached that of free drug.

\section{In vitro drug-release studies}

A dialysis method was applied to investigate the release profile of DOX from DOX-DS nanocomplexes. Figure 5A shows that $14 \%$ of DOX was released during the first 24 hours followed by slow release of $32 \%$ over a time span of 15 days. Figure 5B presents a clearer view of release behavior of DOX-DS nanocomplexes. Furthermore, a controlled release study of free DOX revealed complete diffusion of drug through dialysis membrane within 10 hours (Figure 5C).

\section{Thermal analysis of DOX-DS complexation}

The thermodynamics of DOX-DS interaction was studied by ITC. Analysis by Thermometric Digitam 3 (Figure 6) yielded a binding constant of $0.337 \pm 0.00139 \mathrm{~mol}_{\mathrm{Dox}} / \mathrm{mol}_{\text {Glycosyl }}$ an enthalpy value $(\Delta \mathrm{H})$ of value of $-6.989 \mathrm{kcal} / \mathrm{mol}$, and an entropy value $(\Delta \mathrm{S})$ of $0.011 \mathrm{kcal} / \mathrm{mol}^{\circ} \mathrm{K}$. Free energy $(\Delta \mathrm{G})$ was calculated equal to $-10.267 \mathrm{kcal} / \mathrm{mol}$ by putting $\Delta \mathrm{H}$ and $\Delta \mathrm{S}$ into the Gibbs free energy equation $(\Delta \mathrm{G}=\Delta \mathrm{H}-\mathrm{T} \Delta \mathrm{S})$ (Figure $6 \mathrm{~A})$. 

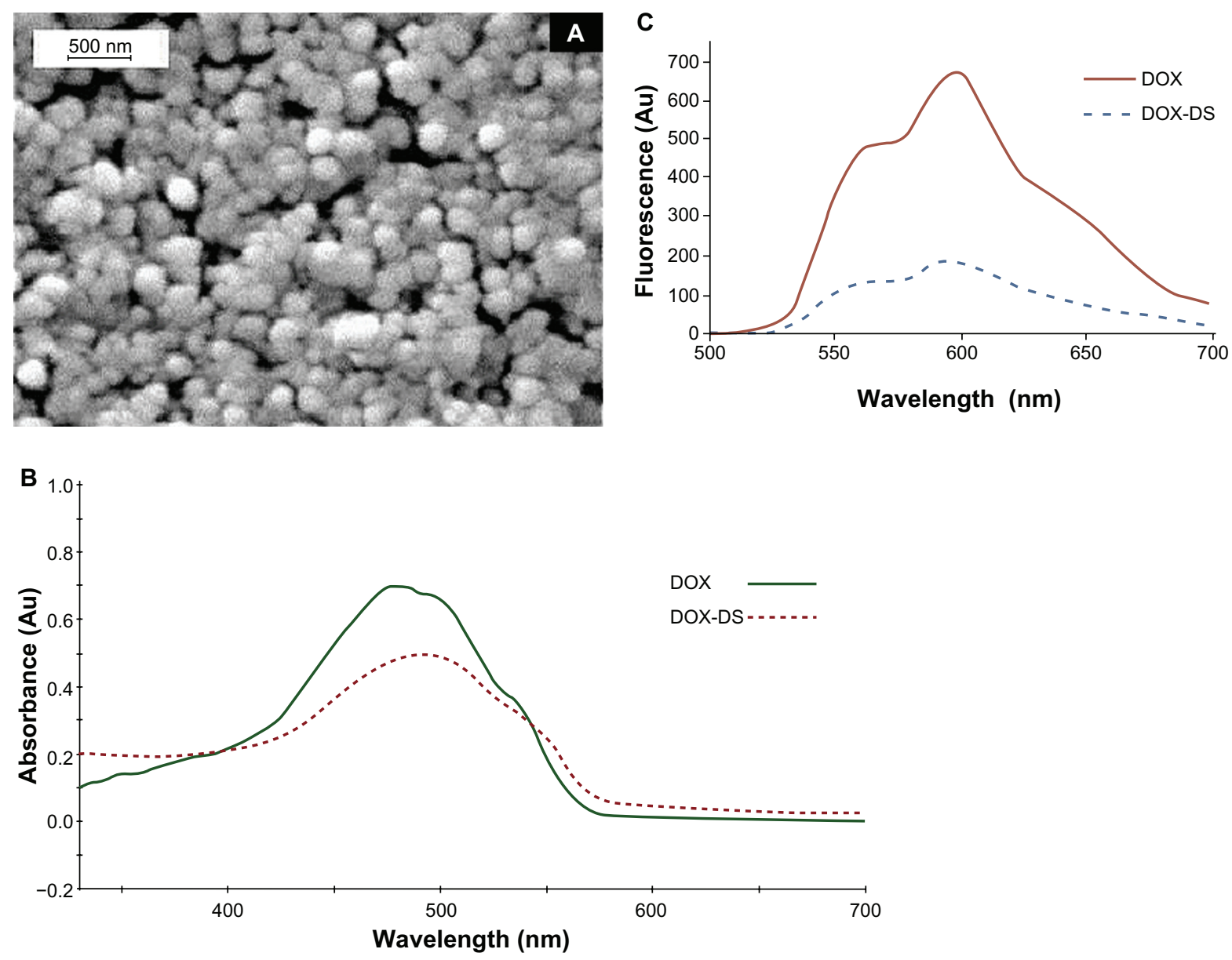

Figure 2 A) Scanning electron micrographs of doxorubicin-dextran sulfate (DOX-DS) nanocomplexes (DOX: $60 \mu \mathrm{g} / \mathrm{mL}$, DS/DOX [w/w]: 0.6), B) UV-visible spectrum of DOX-DS complex and free DOX, and C) fluorescence spectrum of DOX-DS complex and free DOX.

For DOX-DS interaction in the presence of $\mathrm{NaCl} 0.15 \mathrm{M}$, the thermodynamic parameters obtained were: $\Delta \mathrm{H}=-5.37 \mathrm{kcal} / \mathrm{mol}$; $\Delta \mathrm{S}=0.006 \mathrm{kcal} / \mathrm{mol} ; \Delta \mathrm{G}=-7.158 \mathrm{kcal} / \mathrm{mol}$ (Figure $6 \mathrm{~B}$ ); binding constant was $0.757 \pm 0.0061 \mathrm{~mol}_{\text {Dox }} / \mathrm{mol}_{\text {Glycosyl }}$.

\section{Discussion}

The main objective of this study was to study DOX-DS complexation as a potential drug carrier. DOX (Figure 7) is composed of an aglycone moiety to which an amino sugar

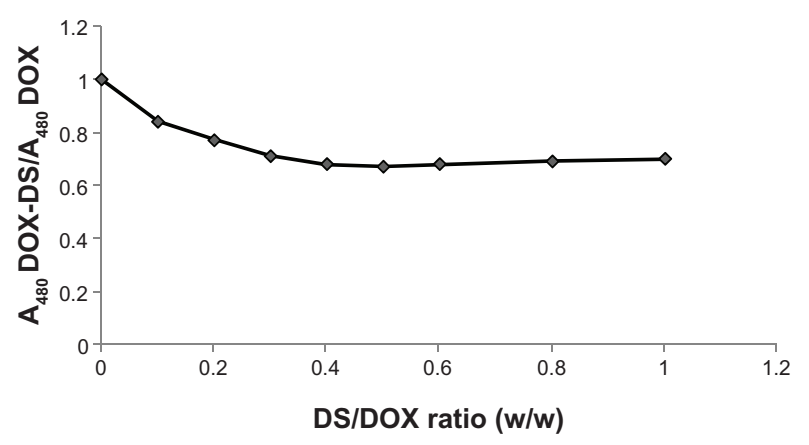

Figure 3 Effect of dextran sulfate/doxorubicin (DS/DOX) ratio on absorbance of DOX-DS complexes. daunosamine is attached via a glycosidic bond. The aglycone part is a tetracyclic chromophore adiamycinone in which the $\mathrm{B}, \mathrm{C}$, and $\mathrm{D}$ rings form a planar anthraquinone system. These aromatic rings result in $\pi$ - $\pi$ stacking properties of DOX, which allows its intercalation into the DNA double helix (one of the mechanisms of action proposed for this anticancer drug). In addition, the amino sugar of DOX has a pKa value of 8.6, which confers alkaline properties onto this molecule and positive charge at neutral $\mathrm{pH}^{44,45}$ Because of the positive charge of DOX, DOX is expected to associate at a high level with polyanionic DS.

The importance of electrostatic interaction in DOX-DS complexation was established by studying the absorption spectrum under different ionic strengths, ie, different concentrations of $\mathrm{NaCl} \mathrm{Na}^{+}$and $\mathrm{Cl}^{-}$shield electrostatic charge of DS and DOX, respectively, and hamper their interaction with one another. At ionic strengths $>0.3 \mathrm{M}$, DOX-DS electrostatic interaction is thoroughly disturbed and the absorption spectrum is not different from that of free DOX. The result is in accordance with that of Kitaeva et al who observed preservation of florescence spectrum of DOX when binding with polyacrylic acid in the presence of $\mathrm{NaCl}^{26} \mathrm{On}$ the other 

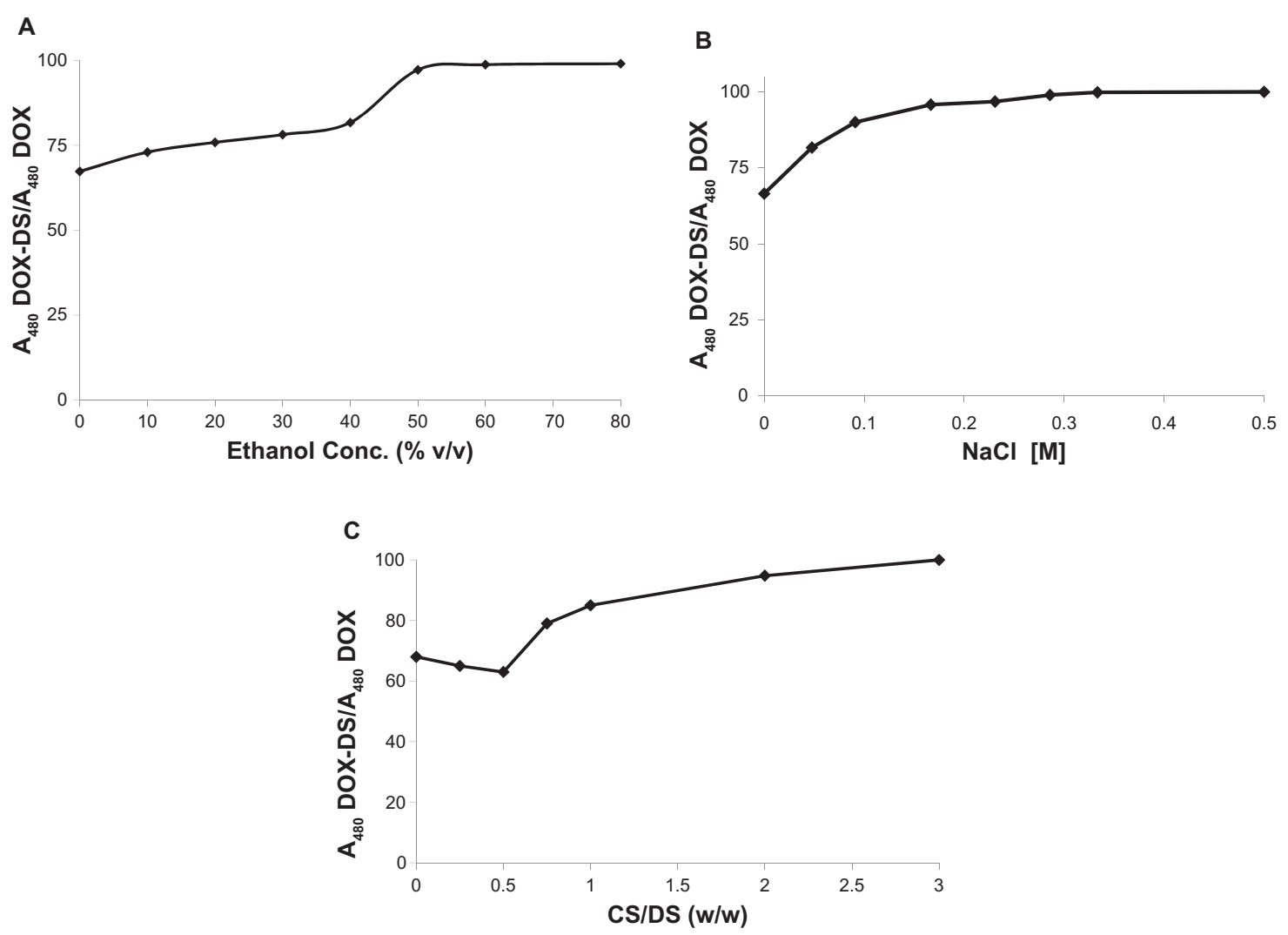

Figure 4 A) Effect of ethanol concentration, B) effect of $\mathrm{NaCl}$ concentration, and C) effect of polycationic chitosan (CS) on absorbance of doxorubicin-dextran sulfate (DOX-DS) nanocomplexes (DOX: $60 \mu \mathrm{g} / \mathrm{mL}$, DS/DOX [w/w]: 0.6).

hand, despite the weakening effect of $\mathrm{NaCl}$ on complexation of DOX with pluronic P85-b-polyacrylic acid block copolymers, Tian et al reported no significant dissociation of DOXpluronic P85-b-poly(acrylic acid) block copolymers. ${ }^{28}$ ITC experiments also demonstrated DOX-DS interaction to be strongly salt-dependent; the enthalpy of binding decreased from $\sim-7.489$ to $\sim-5.37 \mathrm{kcal} / \mathrm{mol}$ in the presence of $\mathrm{NaCl}$ $0.15 \mathrm{M}$. The binding constant also increased from 0.33 to $0.75 \mathrm{~mol}_{\text {Glycosyl }} / \mathrm{mol}_{\text {Dox }}$; the shielding effect of $\mathrm{NaCl}$ weakens the intrarepulsion of the negatively charged sulfate groups and makes the DS structure less extended. As a result, higher numbers of glycosyl units can be accommodated around each DOX molecule leading to a higher $\mathrm{mol}_{\text {Glycosyl }} / \mathrm{mol}_{\text {DOX }}$ ratio.

Furthermore, addition of an H-bond disturbing agent such as ethanol to the system caused recovery of the DOX absorption spectrum. This indicates the contribution of hydrogen bonds to DOX-DX nanocomplexes. According to Tian et al, these hydrogen bonds are ascribed to substituent (HO-C-CO$\mathrm{CH}_{2} \mathrm{OH}$ ) shown by a dashed circle in Figure $7,27,46$ which also takes part in its interaction with DNA. ${ }^{47}$

Addition of DS to DOX resulted in a decrease in the optical density of DOX solution $\left(\lambda_{\max }=480 \mathrm{~nm}\right.$ ) along with a bathochromic shift of about $15 \mathrm{~nm}$. Such alteration in DOX spectra was previously reported in the interaction of DOX with other anionic biopolymers such as DNA, ${ }^{48}$ polyacrylic acid, ${ }^{26}$ and $\gamma$-polyglutamic acid. ${ }^{28}$ Florescence intensity of DOX was also weakened by DS complexation. However, the reduction in florescence was greater than the reduction in $480 \mathrm{~nm}$ absorbance.

In addition, there is a threshold for this hypochromic effect of DS. In fact, complexation of DOX with DS reduces its absorbance to 0.68 with respect to free drug at a DS/DOX ratio of 0.4 ; above this ratio, further addition of DS does not affect absorbance of DOX.

On the other hand, the complexation efficiency curve obtained by the ultracentrifugation method shows a peak rather than a plateau; the curve indicates a maximum association of DOX with DS at a DS/DOX ratio of 0.5. The results reveal that DOX is encapsulated in up to $95 \%$ of DOX-DS complexes up to 0.5 and then declines again at higher DS/DOX ratios. Margaritis et al also reported such high DOX complexation efficiency in DOX- $\gamma$-polyglutamic acid complexes. ${ }^{28}$ This discrepancy between spectroscopy and encapsulation studies may be resolved through dynamic light scattering measurements according to which DOX-DS complexes form particles at a nanoscale level at DS/DOX ratios of 0.4 to 0.6. 
A
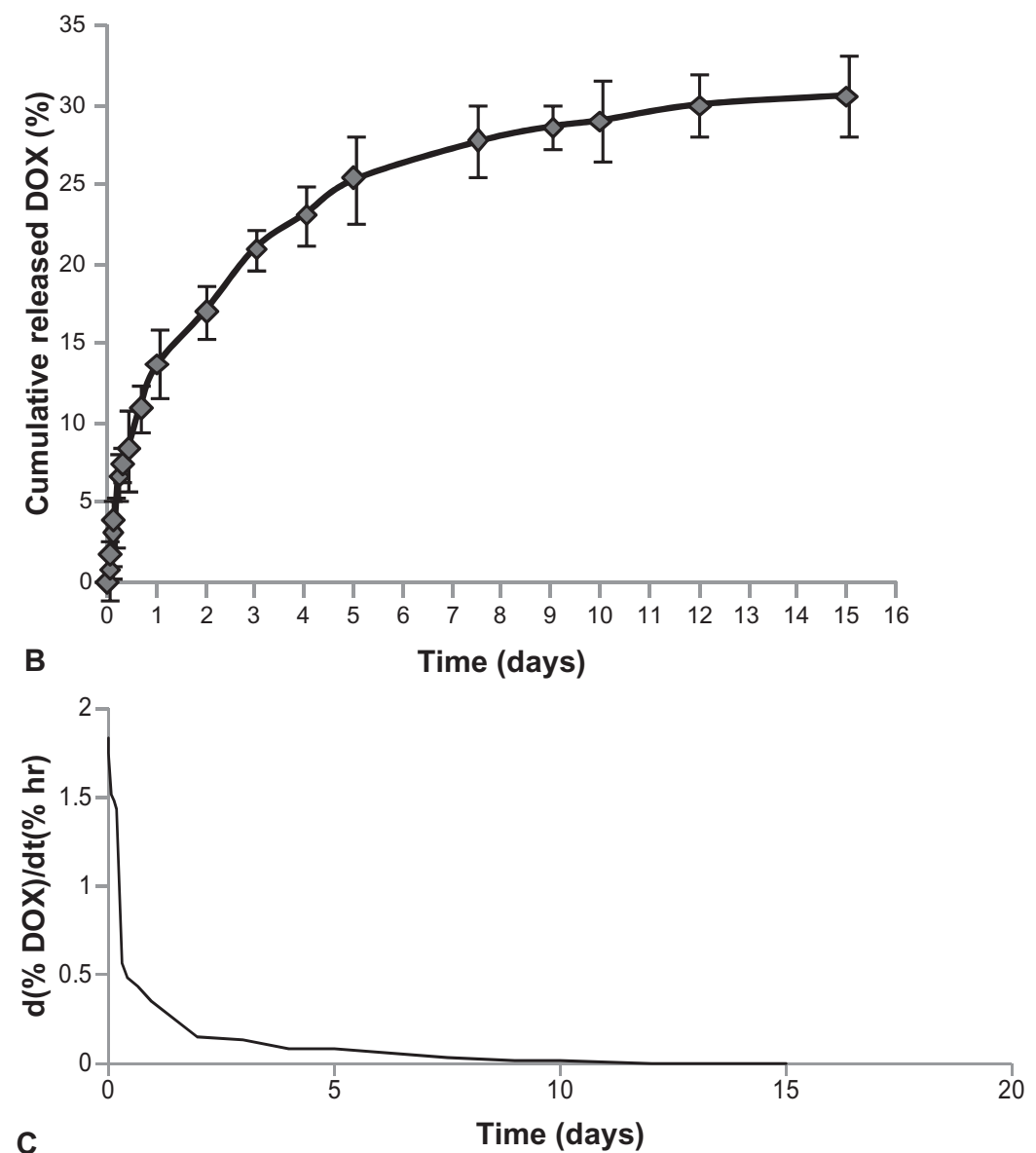

C

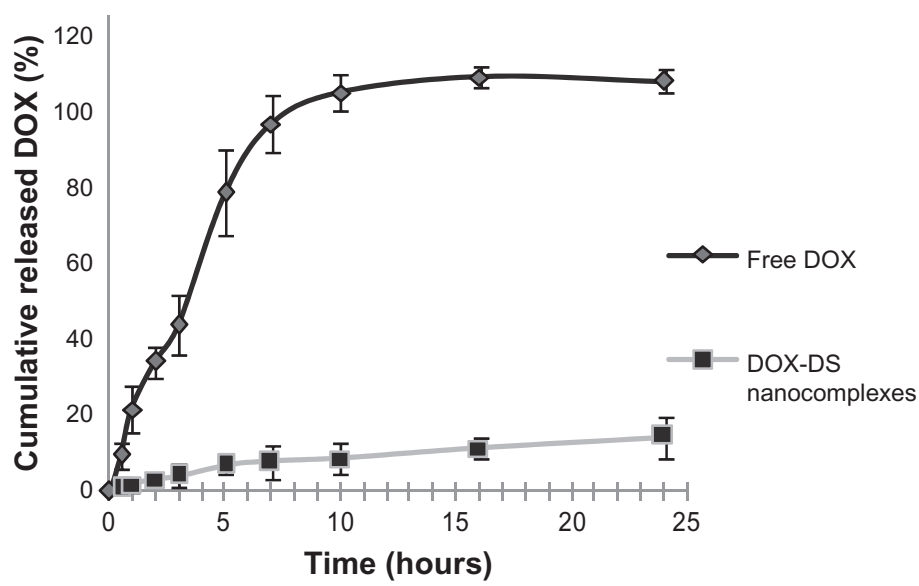

Figure 5 A) In vitro cumulative release and B) in vitro release rate of doxorubicin (DOX) from doxorubicin-dextran sulfate (DOX-DS) nanocomplexes (DOX: $60 \mu \mathrm{g} / \mathrm{mL}$, $\mathrm{DS} / \mathrm{DOX}[\mathrm{w} / \mathrm{w}]: 0.6)(\mathrm{n}=3)$. C) In vitro release of free DOX in phosphate buffer solution $(\mathrm{pH} 7.4), 37^{\circ} \mathrm{C}$.

From all these results, the following mechanism is suggested for DOX-DS complexation: negatively charged DS interacts electrostatically with positively charged DOX molecules, and this interaction is strengthened by hydrogen-bonding interactions between hydroxyl groups of carbohydrate DS and hydroxyl groups attached to the anthraquinone ring in DOX. These interactions bring aromatic-structured DOX molecules in each other's vicinity and facilitate $\pi-\pi$ stacking interactions between them. In fact, DOX molecules are known to form self-associated structures at $[\mathrm{DOX}]>130 \mu \mathrm{M} ;{ }^{49}$ this gives rise to a change in the absorption spectrum of DOX, a phenomenon known as metachromasy. ${ }^{50}$ In the presence of a polyanionic chain such as DS, this metachromatic behavior occurs at lower DOX concentrations. 
A

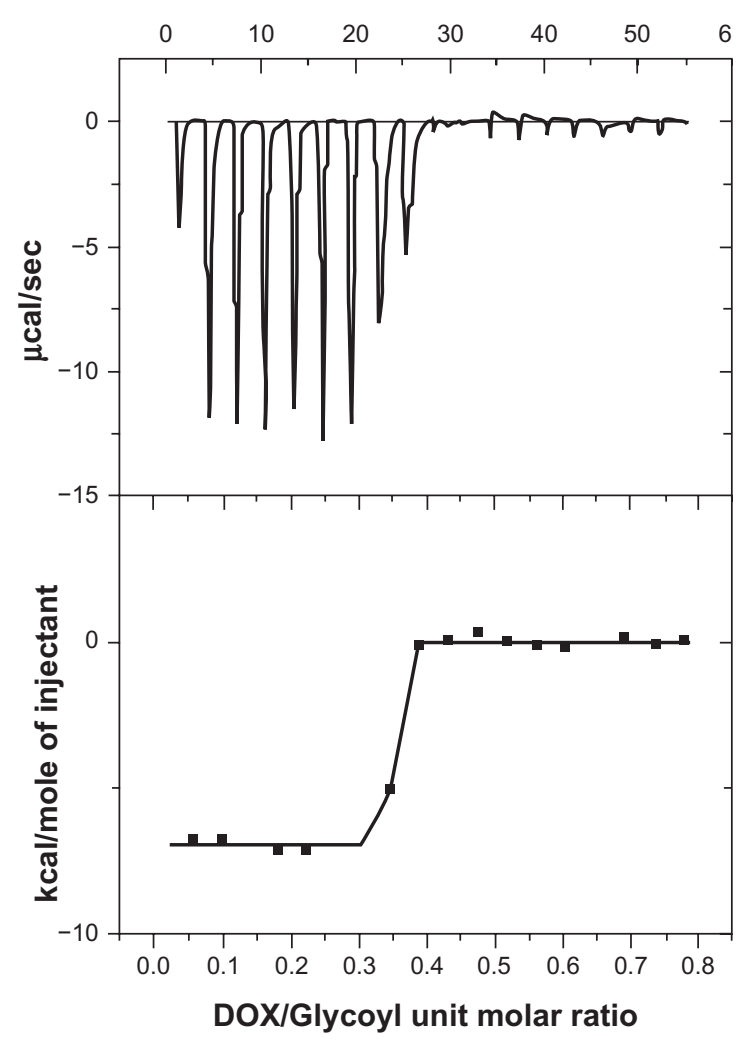

B

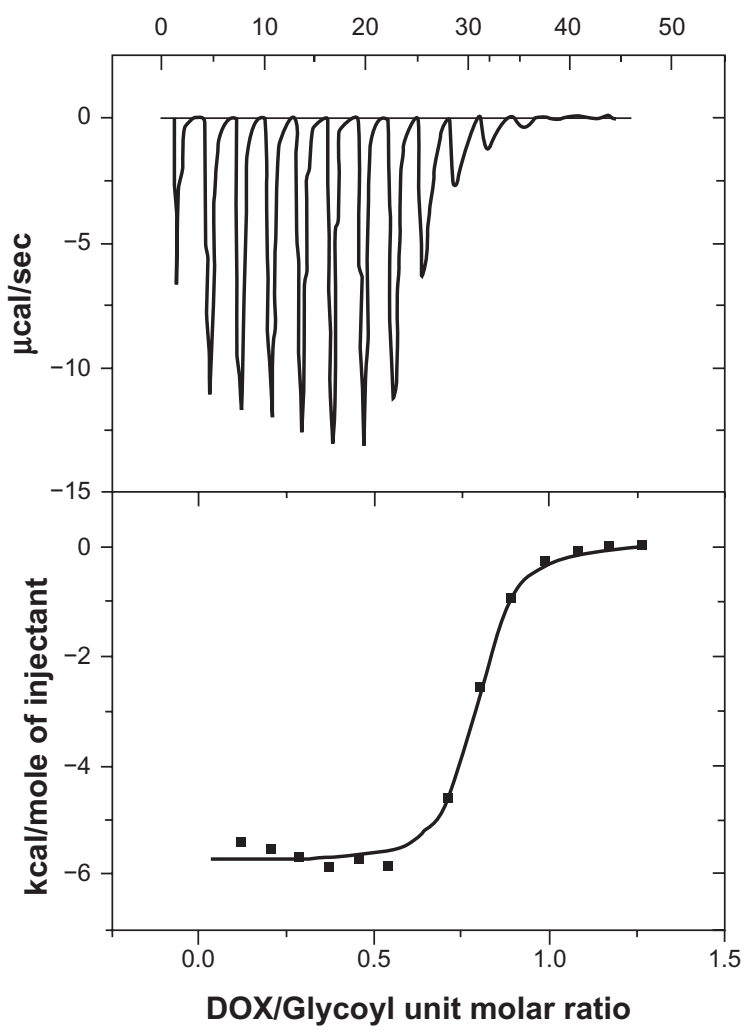

Figure 6 Isothermal titration calorimetry curve obtained from titration of $862 \mu \mathrm{M}$ doxorubicin (DOX) with dextran sulfate (4797 $\mu \mathrm{M}$ glycosyl units) in (A) water (B) NaCl $0.15 \mathrm{M}$.

As DS/DOX ratios increase up to 0.4, more DOX molecules become involved in electrostatic and hydrogen-bonding interactions with DS chains and stacking interactions with each other, and consequently DOX absorbance decreases. At a DS/DOX ratio of 0.4, DOX comes into equilibrium between bulk solution and DOX-DS complexes; DS ratio is high enough to accommodate a maximum fraction of DOX. As illustrated in Figure 8, in the 0.4-0.6 range, a stacked array of DOX molecules acts as a physical crosslinker which, along with

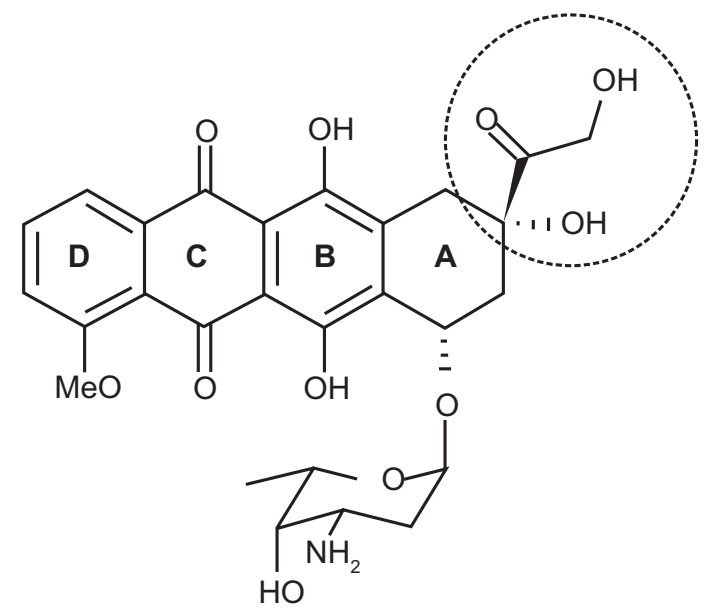

Figure 7 Chemical structure of doxorubicin.
DOX-DS electrostatic interaction, makes DOX-DS complexes compact enough to form drug-polymer nanocomplexes. With further addition of DS to the system at DS/DOX $>0.6$, DOX molecules are distributed among a higher number of DS chains and are positioned farther from each other; stacking interactions are weakened and the DOX-DS network is loosened. Therefore, DOX-DS complexes are not compact enough to form nanocomplexes and are of insufficient density to sediment during ultracentrifugation, which explains the descending part of the encapsulation curve. In fact, a close look at the absorption spectrum of the supernatant of ultracentrifuged DOX-DS complexes reveals the bathometric shift in DOX spectrum at DS/DOX > 0.6; this confirms the presence of DOX-DS complexes not sedimenting by ultracentrifugation.

Thermal analysis of DOX-DS complexation by ITC revealed a binding constant of $0.337 \pm 0.00139 \mathrm{~mol}$ DOX unit per 1 mol DS glycosyl unit; in other words, each DOX molecule binds with 3 DS glycosyl monomers. The ITC curve shows a jump in $\triangle \mathrm{H}$ of system from DOX/glycosyl unit molar ratio $0.25-0.4$, equal to $0.18-0.3 \mathrm{DS} / \mathrm{DOX}(\mathrm{w} / \mathrm{w})$. It seems that a DS/DOX ratio of 0.18 is a critical point where electrostatic interaction is enough to stimulate DOX-DS binding via $\mathrm{H}$-bonding and then stacking interactions, and consequently results in the onset of the exothermic peak. 


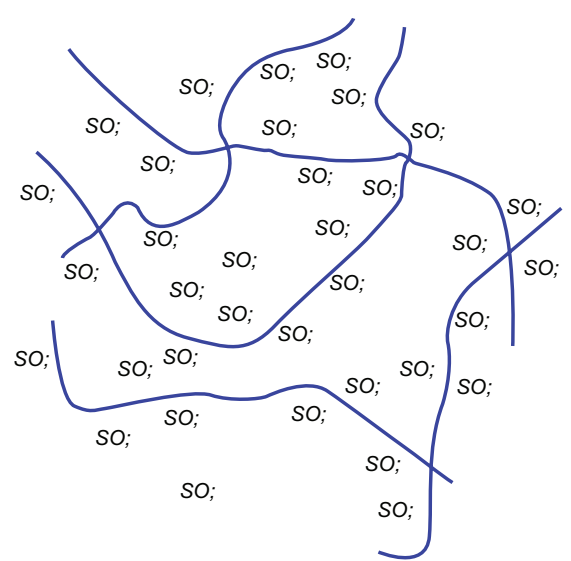

Figure 8 Schematic illustration of doxorubicin-dextran sulfate nanocomplexation.

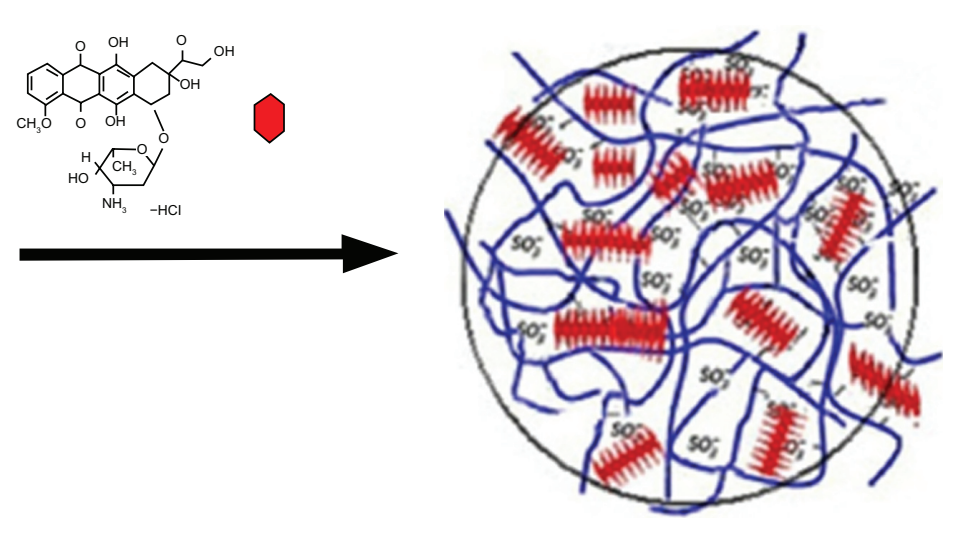

According to the in vitro release study, DOX-DS nanocomplexes release $14 \%$ of DOX at a fast rate during the first 24 hours. This is probably due to release of DOX molecules weakly bound with DS. However, the remaining DOX molecules are tightly complexed to DS by several electrostatic hydrogen-bonding or $\pi-\pi$ interactions; $32 \%$ of this strongly entrapped DOX is released slowly within 2 weeks. Controlled release study of free DOX also demonstrated fast diffusion of drug into dialysate under sink conditions, which proves that diffusion through dialysis tubing (MWCO $12 \mathrm{kDa}$ ) is not a barrier to the passing of the drug, freed from nanocomplexes, into dialysate. Such DOX-DS nanocomplexes may be employed in designing systems for controlled release of DOX. However, in systems dealing with ionic polymers, eg, CS nanoparticles, ${ }^{36,37}$ the competitive effect of the second ionic polymer on DOX-DS interaction should be borne in mind. Contrary to Janes et al, ${ }^{36}$ who observed only partial dissociation of DOX-DS complex in $\mathrm{DS} / \mathrm{DOX}(\mathrm{w} / \mathrm{w}) 10$, our results showed complete resumption of DOX absorbance at $\mathrm{CS} / \mathrm{DOX}(\mathrm{w} / \mathrm{w})>3$, indicating complete dissociation of DOX-DS complexation. The higher charge density of CS and its lower steric hindrance compared with bulky DOX molecules makes it a more favorable electrostatic partner for DS and disrupts DOX-DS complexation.

\section{Conclusion}

DOX-DS nanocomplexes may serve as a drug delivery system with efficient drug encapsulation. Complexes are in the nanoscale range at a DS/DOX $(\mathrm{w} / \mathrm{w})$ ratio of 0.4-0.6. Electrostatic hydrogen bonding and aromatic interactions play the main roles in their formation. The DOX release profile of nanocomplexes shows an initial fast release followed by a sustained release. DOX-DS interaction is affected by other anionic polymers, an important point when applying DS in order to enhance DOX encapsulation into other polymeric delivery systems.

\section{Disclosure}

The authors declare no conflicts of interest.

\section{References}

1. Hoskins WJ, Perez CA, Young RC, Barakat RR, Markman M. Principles and Practice of Gynecologic Oncology. 4th ed. Lippincott Williams \& Wilkins; 2005.

2. Hrushesky WJM. Bone marrow suppression from doxorubicin and cis-diamminedichloroplatinum is substantially dependent upon both circadian and circannual stages of administration. Ann N York Acad Sci. 1982;397:293-295.

3. Swain SM, Whaley FS, Ewer MS. Congestive heart failure in patients treated with doxorubicin: A retrospective analysis of three trials. Cancer. 2003;97(11):2869-2879.

4. Schwartz RG, McKenzie WB, Alexander J, et al. Congestive heart failure and left ventricular dysfunction complicating doxorubicin therapy: Seven-year experience using serial radionuclide angiocardiography. Am J Med. 1987;82(6):1109-1118.

5. Von Hoff DD, Layard MW, Basa P. Risk factors for doxorubicin-induced congestive heart failure. Ann Intern Med. 1979;91(5):710-717.

6. Zijlstra JG, De Vries EGE, Mulder NH. Multifactorial drug resistance in an Adriamycin-resistant human small cell lung carcinoma cell line. Cancer Res. 1987;47(7):1780-1784.

7. Kaye S, Merry S. Tumour cell resistance to anthracyclines: A review. Cancer Chemother Pharmacol. 1985;14(2):96-103.

8. Nair S, Singh SV, Samy TSA, Krishan A. Anthracycline resistance in murine leukemic P388 cells. Role of drug efflux and glutathione related enzymes. Biochem Pharmacol. 1990;39(4):723-728.

9. Bradley G, Juranke PF, Ling V. Mechanism of multidrug resistance. Biochim Biophys Acta - Reviews on Cancer. 1988;948(1):87-128.

10. Patil RR, Guhagarkar SA, Devarajan PV. Engineered nanocarriers of doxorubicin: a current update. Crit Rev Ther Drug Carrier Syst. 2008; 25(1):1-61.

11. Gil ES, Li J, Xiao H, Lowe TL. Quaternary ammonium $\hat{I}^{2}$-cyclodextrin nanoparticles for enhancing doxorubicin permeability across the in vitro blood-brain barrier. Biomacromolecules. 2009;10(3):505-516.

12. Subedi RK, Kang KW, Choi HK. Preparation and characterization of solid lipid nanoparticles loaded with doxorubicin. Eur J Pharm Sci. 2009;37(3-4):508-513.

13. Missirlis D, Kawamura R, Tirelli N, Hubbell JA. Doxorubicin encapsulation and diffusional release from stable, polymeric, hydrogel nanoparticles. Eur J Pharm Sci. 2006;29(2):120-129.

14. Park J, Fong PM, Lu J, et al. PEGylated PLGA nanoparticles for the improved delivery of doxorubicin. Nanomed. 2009;5(4):410-418.

15. Atyabi F, Talaie F, Dinarvand R. Thiolated chitosan nanoparticles as an oral delivery system for amikacin: In vitro and ex vivo evaluations. J Nanosci Nanotechnol. 2009;9(8):4593-4603. 
16. Esmaeili F, Dinarvand R, Ghahremani MH, et al. Docetaxel-albumin conjugates: Preparation, in vitro evaluation and biodistribution studies. J Pharm Sci. 2009;98(8):2718-2730.

17. Saremi S, Atyabi F, Akhlaghi SP, Ostad SN, Dinarvand R. Thiolated chitosan nanoparticles for enhancing oral absorption of docetaxel: Preparation, in vitro and ex vivo evaluation. Int J Nanomedicine. 2011;6(1): 119-128.

18. Akhlaghi SP, Saremi S, Ostad SN, Dinarvand R, Atyabi F. Discriminated effects of thiolated chitosan-coated pMMA paclitaxel-loaded nanoparticles on different normal and cancer cell lines. Nanomedicine. 2010;6(5):689-697.

19. Majzoob S, Atyabi F, Dorkoosh F, Kafedjiiski K, Loretz B, BernkopSchnürch A. Pectin-cysteine conjugate: Synthesis and in-vitro evaluation of its potential for drug delivery. J Pharm Pharmacol. 2006; 58(12):1601-1610.

20. Lee ES, Na K, Bae YH. Doxorubicin loaded $\mathrm{pH}$-sensitive polymeric micelles for reversal of resistant MCF-7 tumor. $J$ Control Release. 2005;103(2):405-418

21. Verdière AC, Dubernet C, Némati F, Poupon MF, Puisieux F, Couvreur P. Uptake of doxorubicin from loaded nanoparticles in multidrug-resistant leukemic murine cells. Cancer Chemother Pharmacol. 1994;33(6):504-508.

22. De Verdière AC, Dubernet $\mathrm{C}$, Némati F, et al. Reversion of multidrug resistance with polyalkylcyanoacrylate nanoparticles: Towards a mechanism of action. Br J Cancer. 1997;76(2):198-205.

23. Wong HL, Bendayan R, Rauth AM, Xue HY, Babakhanian K, Wu XY. A mechanistic study of enhanced doxorubicin uptake and retention in multidrug resistant breast cancer cells using a polymer-lipid hybrid nanoparticle system. J Pharmacol Exp Ther. 2006;317(3): 1372-1381.

24. Ehtezazi T, Govender T, Stolnik S. Hydrogen bonding and electrostatic interaction contributions to the interaction of a cationic drug with polyaspartic acid. Pharm Res. 2000;17(7):871-877.

25. Govender T, Ehtezazi T, Stolnik S, Illum L, Davis SS. Complex formation between the anionic polymer (PAA) and a cationic drug (procaine $\mathrm{HCl}$ ): characterization by microcalorimetric studies. Pharm Res. 1999;16(7):1125-1131.

26. Kitaeva MV, Melik-Nubarov NS, Menger FM, Yaroslavov AA. Doxorubicin-poly(acrylic acid) complexes: interaction with liposomes. Langmuir. 2004;20(16):6575-6579.

27. Tian Y, Bromberg L, Lin SN, Alan Hatton T, Tam KC. Complexation and release of doxorubicin from its complexes with pluronic P85-b-poly(acrylic acid) block copolymers. J Control Release. 2007;121(3):137-145.

28. Margaritis A, Manocha B. Controlled release of doxorubicin from doxorubicin $/ \hat{\mathrm{I}}^{3}$-polyglutamic acid ionic complex. Journal of Nanomaterials. 2010:1-8.

29. Pratesi G, Savi G, Pezzoni G. Poly-L-aspartic acid as a carrier for doxorubicin: A comparative in vivo study of free and polymer-bound drug. Br J Cancer. 1985;52(6):841-848.

30. Bogush T, Smirnova G, Shubina I, Syrkin A, Robert J. Direct evaluatlon of intracellular accumulation of free and polymer-bound anthracyclines. Cancer Chemother Pharmacol. 1995;35(6):501-505.

31. Kwon GS, Yokoyama M, Okano T, Sakurai Y, Kataoka K. Biodistribution of micelle-forming polymer-drug conjugates. Pharm Res. 1993;10(7):970-974.
32. Jeong Y-I, Nah J-W, Lee H-C, Kim S-H, Cho C-S. Adriamycin release from flower-type polymeric micelle based on star-block copolymer composed of poly([gamma]-benzyl-glutamate) as the hydrophobic part and poly(ethylene oxide) as the hydrophilic part. Int J Pharm. 1999;188(1):49-58.

33. Kataoka K, Matsumoto T, Yokoyama M, et al. Doxorubicin-loaded poly(ethylene glycol)-poly([beta]-benzyl - aspartate) copolymer micelles: their pharmaceutical characteristics and biological significance. J Control Release. 2000;64(1-3):143-153.

34. Oh I, Lee K, Kwon HY, et al. Release of adriamycin from poly( $\mathrm{I}^{3}-$ benzyl-L-glutamate)/poly(ethylene oxide) nanoparticles. Int J Pharm. 1999;181(1):107-115.

35. Chen Y. Synthesis of albumin-dextran sulfate microspheres possessing favourable loading and release characteristics for the anticancer drug doxorubicin. J Control Release. 1994;31(1):49-54.

36. Janes KA, Fresneau MP, Marazuela A, Fabra A, Alonso MJ. Chitosan nanoparticles as delivery systems for doxorubicin. J Control Release. 2001;73(2-3):255-267.

37. Tan ML, Friedhuber AM, Dunstan DE, Choong PFM, Dass CR. The performance of doxorubicin encapsulated in chitosan-dextran sulphate microparticles in an osteosarcoma model. Biomaterials. 31(3):541-551.

38. Yang M, Chan HL, Lam W, Fong WF. Cytotoxicity and DNA binding characteristics of dextran-conjugated doxorubicins. Biochimica et Biophysica Acta - General Subjects. 1998;1380(3):329-335.

39. Shinozawa S, Fukuda T, Araki Y, Oda T. Effect of dextran sulfate on the survival time and mitochondrial function of adriamycin (doxorubicin)treated mice. Toxicol Appl Pharmacol. 1985;79(2):353-357.

40. Bisht S, Maitra A. Dextran-doxorubicin/chitosan nanoparticles for solid tumor therapy. Wiley Interdisciplinary Reviews: Nanomedicine and Nanobiotechnology. 2009;1(4):415-425.

41. Lam W, Chan H, Yang M, Cheng S, Fong W. Synergism of energy starvation and dextran-conjugated doxorubicin in the killing of multidrugresistant KB carcinoma cells. Anticancer Drugs. 1999;10(2):171-178.

42. Lam W, Leung $\mathrm{CH}$, Chan HL, Fong WF. Toxicity and DNA binding of dextran-doxorubicin conjugates in multidrug-resistant KB-V1 cells: optimization of dextran size. Anticancer Drugs. 2000;11(5):377-384.

43. Sheldon K, Marks A, Baumal R. Sensitivity of multidrug resistant $\mathrm{KB}-\mathrm{C} 1$ cells to an antibody-dextran-adriamycin conjugate. Anticancer Res. 1989;9(3):637-641.

44. Angerer J, Schaller K-H. Analyses of Hazardous Substances in Biological Materials. Vol 7. Weinheim: Wiley-VCH; 2001.

45. Rubin SC. Chemotherapy of Gynecologic Cancers: Society of Gynecologic Oncologists Handbook. 2nd ed. Baltimore: Lippincott Williams \& Wilkins; 2003.

46. Tian Y, Ravi P, Bromberg L, Hatton TA, Tam KC. Synthesis and aggregation behavior of pluronic F87/poly(acrylic acid) block copolymer in the presence of doxorubicin. Langmuir. 2007;23(5):2638-2646.

47. Chaires JB, Satyanarayana S, Suh D, Fokt I, Przewloka T, Priebe W. Parsing the free energy of anthracycline antibiotic binding to DNA. Biochemistry. 1996;35(7):2047-2053.

48. Hajian R, Shams N, Mohagheghian M. Study on the interaction between doxorubicin and deoxyribonucleic acid with the use of methylene blue as a probe. J Braz Chem Soc. 2009;20(8):1399-1405.

49. Michaelis L, Granick S. Metachromasy of basic dyestuffs. J Am Chem Soc. 1945;67(7):1212-1219.

50. Krishnamurthy KV. Methods in Cell Wall Cytochemistry. LLC: CRC Press; 1999

International Journal of Nanomedicine

\section{Publish your work in this journal}

The International Journal of Nanomedicine is an international, peerreviewed journal focusing on the application of nanotechnology in diagnostics, therapeutics, and drug delivery systems throughout the biomedical field. This journal is indexed on PubMed Central, MedLine, CAS, SciSearch $\AA$, Current Contents $\AA /$ Clinical Medicine,

Journal Citation Reports/Science Edition, EMBase, Scopus and the Elsevier Bibliographic databases. The manuscript management system is completely online and includes a very quick and fair peer-review system, which is all easy to use. Visit http://www.dovepress.com/ testimonials.php to read real quotes from published authors. 